\title{
The Second Byurakan Survey - The Final Results. The Multiwavelength AGN Survey in the SBS Area
}

\author{
J.A. Stepanian \\ Instituto de Astronomia, Universidad Nacional Autonoma de México, \\ A.P. 70-264, México D.F. 04510, México. \\ V.H. Chavushyan \\ Instituto Nacional de Astrofísica Optica y Electrónica, A.P. 51 y 216, \\ C.P. 72000, Puebla, Pue., México.
}

\begin{abstract}
.
On the basis of the Second Byurakan Survey (SBS) we have produced the largest and most homogeneous new complete samples of faint Markarian galaxies, Sy galaxies and bright QSOs. The General Catalogue of the SBS Survey, which is completed and prepared for publication, contains the data for more than 3600 objects, half of which are star-like objects while the other half are galaxies. Nearly 2500 slit spectra were obtained for about 2100 SBS objects. 561 new QSOs and AGNs in the magnitude range $15.5<B<19.5$ with redshifts $0.0<z<3.2$ were spectroscopically confirmed. A sample of 17 BLL has been isolated as well.

The volume for reliable investigation of faint Markarian UVX galaxies has been extended out to a distance of about $\sim 500 \mathrm{Mpc}$, more than 50 times deeper than in the FBS. Faint Markarian UVX galaxies from the SBS survey comprise $\sim 12 \%$ of field galaxies, slightly greater than in the First Markarian survey; $\sim 10 \%$ of them $(\sim 120)$ turned out to be Sy galaxies.

A complete sample of faint $(B \leq 17.0, z \leq 0.1)$ Sy galaxies has been constructed. In the spatial volume with radius of about $500 \mathrm{Mpc}$, the relative number of AGNs is $1.2 \%$ of field galaxies. One of the most complete and representative samples of bright QSOs $(\sim 130)$ in the magnitude range $15.7<B \leq 17.5$ with redshifts $0.3 \leq z \leq 2.2$ has been constructed as well.

The objects in the combined complete sample of SBS Sy galaxies and QSOs might be roughly divided into two groups: classical Sy galaxies and QSOs in agreement with the previous classification scheme and nonclassical SyG and QSOs for which the existing classification scheme is not sufficient for their classification. The presence of narrow line QSOs (NLQSOs) or so-called QSO II type objects is established. There is a continuous transition of all properties between all types of Sy galaxies and QSOs, from Liners through Sy2, NLSy1s, BLSy1 to NLQSOs and BLQSOs.

Preliminary results from the Multiwavelength AGN Survey (MWAGN) in X-ray, Radio and IR wavebands in the SBS area are presented. The MWAGN survey in the SBS area shows that the ROSAT or FIRST sur-
\end{abstract}


veys by themselves cannot represent the whole AGN/QSO sample. The ROSAT Bright sources along with the FIRST sources comprise only $25 \%$ of the whole SBS QSO sample. The IRAS survey is effective for a redshift less than $z<0.05$. All classical Sy2s but only $\sim 50 \%$ of classical Syls are IRAS sources. Luminous and Ultraluminous IRAS SBS AGNs comprise $\sim 25 \%$ of the whole AGN sample. The greater part of SBS AGN/QSOs are neither ROSAT nor FIRST nor IRAS LIG or ULIG sources. They are optically emitting AGN/QSOs.

The multiwavelength "complete" sample of bright QSOs in the SBS area allows us to conclude that the fast evolution of bright QSOs is more likely the result of a selection effect than the result of fast cosmological evolution. The $\operatorname{LogN}-\mathrm{B}$ relation for these QSOs reduces to the value $\log N-B=0.67 \pm 0.005$.

A direct empirical estimate of the effect of overlapping images is obtained from the MWAGN survey. The effect of overlap comprises $~ 5 \%$ for QSOs brighter than $B \leq 17.0$ and $\sim 10 \%$ for QSOs brighter than $B \leq 17.5$.

\section{Introduction}

The tremendous success of the Markarian survey initiated a number of other extragalactic thin-prism surveys and initiated a new direction in extragalactic astronomy-the systematic search for peculiar objects using low-dispersion spectroscopy.

J.Stepanian started his work on the First Byurakan Survey in 1974 in Byurakan Observatory. At the end of 1974 in parallel with the FBS, we undertook a new survey -the Second Byurakan Survey. V. Chavushyan joined the SBS survey in 1986. 27 years after the beginning of the SBS survey we may report: Beniamin Egishevich Markarian, the complete sample of faint SBS Sy galaxies and QSOs is compiled. Your rule of life, the exactitude and the scrupulousness regarding any question, especially in the field of your main work of the First Byurakan Survey, were continued in the Second Byurakan Survey. A short summary of today's state of the SBS survey, and a brief description of the Byurakan surveys are presented below.

\section{Byurakan Surveys (FBS and SBS)}

The First Byurakan Survey (FBS). Begining in the mid-1960s and continuing through 1980, the first large-scale objective-prism survey for galaxies with blue and UVX in their continuum radiation was conducted by Markarian. The observatons of the First Byurakan survey, also commonly known as the Markarian survey, were carried out with the famous Byurakan 1-m Schmidt telescope with the use of a low-dispersion $(1800 \AA / \mathrm{mm}$ at $\mathrm{H} \gamma), 1.5 \mathrm{deg}$ objective prism. More than 2500 photographic plates which covered nearly $17000 \mathrm{deg}^{2}$ were obtained. The FBS was completed in 1980 and published in a series of 15 papers (Markarian 1967, Markarian et al. 1981). 
The 1500 Markarian galaxies contained in the FBS have provided the principal base from which the major types of AGNs have been discovered, classified, and studied in detail by numerous workers. The FBS resulted in a complete sample of AGNs down to a limiting magnitude 15.2 . Markarian galaxies comprise $10 \%$ of field galaxies, and about $10 \%$ of Markarian galaxies turned out to be Sy galaxies, so $1 \%$ of field galaxies were found to be Sy galaxies. The FBS remains perhaps the best known source of AGN in the Local Universe.

The Second Byurakan Survey (SBS). The SBS survey, which is the continuation of the Markarian survey, was aimed to reach fainter limiting magnitudes. The primary goal of the SBS survey was to extend the Markarian survey as deep as possible to obtain a large, well-defined sample of AGNs and QSOs that were selected in a reasonably uniform fashion.

The SBS started in 1974, and plate searching was completed in 1991. A total area of $1000 \mathrm{deg}^{2}$ within the contiguous strip defined by $7^{h} 40^{m}<\alpha<17^{h} 15^{m}$, $+49^{\circ}<\delta<+61^{\circ}$ has been observed. The SBS was conducted with the sarne $40-$ 52 inch Schmidt telescope, but with the combination of a set of three objective prisms, 1.5, 3 and 4 degree, respectively, in combination with more modern (in 1974) IIIaJ and IIIaF emulsions sensitized in heated nitrogen. The use of both emulsions extends the wavelength range of sensitivity, increases the uniformity of discoveries, and permits the acquisition of spectra for AGNs down to $B \sim 19.5$, which is about 2-3 magnitudes fainter than was achieved in the FBS.

The SBS survey area is covered by 65 contiguous fields with a size of $4 \times 4$ degrees (plate size $16 \times 16 \mathrm{~cm}$ ), which were obtained in three strips along R.A. with centers of $+59,+55$ and +51 degrees. To increase the effectiveness and homogeneity of the survey, all the fields were covered by sets of two to five objective prism plates with IIIaF +1.5 degree prism $(3500-7500 \AA)$ and IIIaJ +1.5 degree prism (3500-5400 $\AA$ ). For the main part of them, sets of two to five objective prism plates with IIIaJ+3 degree prism+GG495 filter $(4950-5400 \AA)$, and IIIaF+4 degree prism+RG610 filter $(6300-7000 \AA)$ were obtained. Some fields were covered with IV-N+4 degree prism+RG8 filter (6900-8500 $\AA$ ). As a result, the red, blue-green and UV parts of the spectra with practically identical spectral resolution $(\sim 1000 \AA)$ were available for reliable investigation. The SBS plates were scanned by eye and candidates were selected on the basis of evidence of a strong UV continuum, emission features, or unusual spectral energy distribution. A selection of $\sim 3600$ objects of which 1800 galaxies $(\sim 1000 \mathrm{UVX}$ and $\sim 800$ ELG without significant UV excess) and 1800 stellar objects with excess ultraviolet emission is the main result of the SBS survey.

Medium-resolution spectral observations $(R \sim 5-10 \AA$ and $S / N \sim 15-25)$ were obtained for about 2100 SBS objects from 1977 to 2001 . The spectroscopic and photometric observations were made on the 6-m and 1-m telescopes of the SAO (Russia) on the 4.5-m MMT (USA), the 2.6-m Byurakan (Armenia) and 2.1-m GHO (Mexico) telescopes.

The first list of SBS objects was published in 1983 (Markarian \& Stepanian 1983). In total there are seven lists published (Stepanian 1994, and references therein). The main parameters for all studied objects were published in a series of papers (Markarian \& Stepanian 1984a,b; Markarian et.al. 1985, 1986; Stepanian et.al. 1988-2001, Stepanian 1994- 1998). 
So far, the identifications of 578 new AGN/QSOs, $\sim 1000$ galaxies with narrow emission lines and $\sim 1000$ galactic stars, the vast majority of which are hot WD and subdwarfs, have been spectroscopically confirmed in a area of $\sim 1000 \mathrm{deg}^{2}$ of the SBS survey. The present status of the SBS survey is illustrated in Table 1.

Table 1. The Second Byurakan Survey. Present (2001) Status

\begin{tabular}{|c|c|c|c|c|c|}
\hline Spectroscopy & \multicolumn{2}{|c|}{ Total $\sim 2500$ spectra } & \multicolumn{2}{|c|}{ Photometry $\sim 500$} & \\
\hline \multicolumn{2}{|c|}{ SBS Stellar $\sim 1250 \mathrm{sp}$} & \multicolumn{2}{|c|}{ SBS QSOs $N=441$} & \multicolumn{2}{|c|}{ SBS stars $N=810$} \\
\hline SBS QSOs & Number & Other source & Number & SBS stars & Number \\
\hline BALQSO & 15 & Radio & 200 & WD & $\sim 300$ \\
\hline DampQSO & 5 & X-ray & 200 & subdwarf & $\sim 200$ \\
\hline Ly forest & 15 & Gamma-ray & 2 & $\mathrm{HBB}+\mathrm{NHB}$ & $\sim 100$ \\
\hline BLLac & 17 & BLAZAR & 3 & $\mathrm{~F} / \mathrm{G}$ & $\sim 100$ \\
\hline Abs. QSOs & 35 & IRAS & 20 & Continual & 27 \\
\hline Grav. lenses & 3 & & & $\mathrm{C} 2+\mathrm{dMe}$ & 45 \\
\hline Other & 360 & & & $\mathrm{CV}$ & 25 \\
\hline & & & & Composite & 17 \\
\hline \multicolumn{2}{|c|}{ SBS Galaxies $\sim 1250$ sp. } & \multicolumn{2}{|c|}{ SBS SyG N $=120$} & \multicolumn{2}{|c|}{ Classified $\sim 800$} \\
\hline SBS SyG & Number & Classified & Number & Other sour. & Number \\
\hline Classical SyG & 62 & Liners & 97 & IRAS & $\sim 600$ \\
\hline Sy1 & 26 & SBN & 350 & Radio & $\sim 200$ \\
\hline Sy1.5 & 8 & $\mathrm{BCDG}$ & 130 & X-ray & $\sim 50$ \\
\hline Sy2 & 28 & HII & 100 & Close-bin. & $\sim 250$ \\
\hline Non-class.SyG & 58 & ELG & 600 & & \\
\hline NLSy1 & 40 & Abs.Gal & 20 & & \\
\hline Sy/Lnr & 18 & & & & \\
\hline
\end{tabular}

The subsample of relatively bright $B \leq 17.5$ stellar objects $(\mathrm{N}=820)$ was investigated completely. $\mathrm{B}$ and $\mathrm{V}$ band $\mathrm{CCD}$ photometric measurements were made for all $B \leq 17.5$ spectroscopically-confirmed new SBS QSOs. A complete sample of 182 optically selected SBS QSOs was isolated.

A similar investigation was done for the SBS galaxy sample. Slit spectra were obtained for $\sim 800$ galaxies with $B \leq 17.0$ which comprise nearly $70 \%$ of the completeness level. A complete sample of faint SBS Sy galaxies was isolated. $\mathrm{B}$ and $\mathrm{V}$ band $\mathrm{CCD}$ photometric measurments were made for nearly $80 \%$ of them.

We have produced the largest and most homogeneous, new combined complete optical sample of SBS QSOs and Sy galaxies, which comprises $\sim 280$ objects in an area of $1000 \mathrm{deg}^{2}$ of the Northern sky. This is perhaps the first deep $(z \sim 0.15)$ representative sample of faint AGNs compiled in a uniform and homogeneous fashion.

\section{The Complete Optical Samples of SBS AGN/QSOs}

The total SBS AGN/QSO optical sample contains 578 objects (120 SyG, 441 QSO and $17 \mathrm{BLL}$ ) in the magnitude range $15.5<B<19.5$ and redshift range 

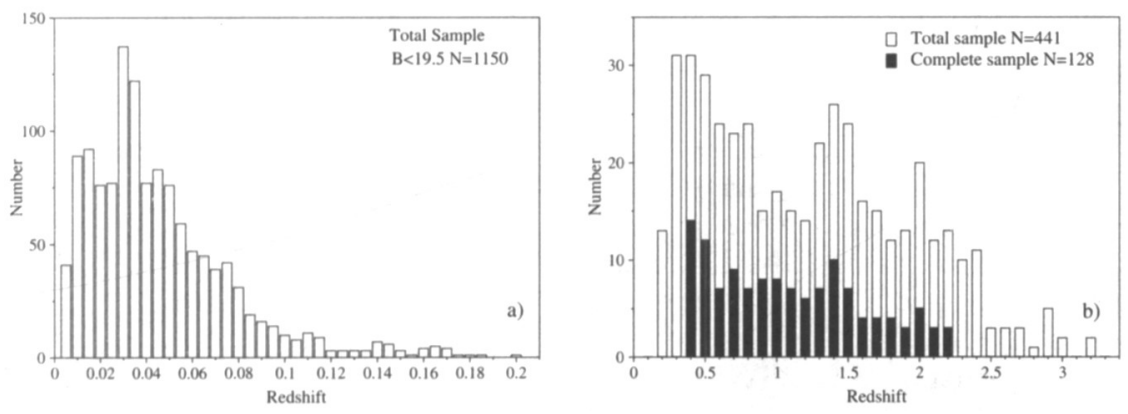

Figure 1. Redshift distribution: a) SBS Galaxies, b) SBS QSOs.

$0.0<z<3.2$ (75 with $0.0<z \leq 0.1,75$ with $0.1<z<0.3$, 373 with $0.3 \leq z \leq 2.2$ and 38 with $2.2<z<3.2$ ). We divide the sample of SBS AGN/QSOs into two groups by redshift and magnitude where the subsample of isolated objects is methodologically complete.

\subsection{The Complete Sample of SBS Sy Galaxies. Magnitude Range 13.0 $<\mathrm{B} \leq \mathbf{1 7 . 0 ,}$ Redshift Range $0.0<\mathrm{z} \leq \mathbf{0 . 1}$}

The surface density of SBS galaxies corresponds to a value of $\sim 1.84$ galaxy per $d e g^{2}$ to a limit of $B \sim 19$. The more precise value of the surface density of SBS UVX, ELG without UV continua and SBS Sy galaxies as a function of limiting magnitude is presented in Table 2.

Table 2. The surface density of SBS UVX, ELG and SyG

\begin{tabular}{|l|l|l|l|l|l|l|l|}
\hline & Total & \multicolumn{7}{|c|}{ Surface density } \\
\cline { 3 - 8 } & number & $B \leq 15.0$ & $B \leq 15.5$ & $B \leq 16.0$ & $B \leq 16.5$ & $B \leq 17.0$ & $B \leq 19.0$ \\
\hline SBS total & $\mathbf{1 8 4 0}$ & $\mathbf{0 . 1 2}$ & $\mathbf{0 . 2 7}$ & $\mathbf{0 . 5 3}$ & $\mathbf{0 . 8 8}$ & $\mathbf{1 . 2 5}$ & $\mathbf{1 . 8 4}$ \\
SBS UVX & $\mathbf{1 0 1 0}$ & $\mathbf{0 . 0 7}$ & $\mathbf{0 . 1 3}$ & $\mathbf{0 . 2 7}$ & $\mathbf{0 . 4 9}$ & $\mathbf{0 . 7 2}$ & $\mathbf{1 . 0 1}$ \\
SBS ELG & $\mathbf{8 3 0}$ & $\mathbf{0 . 0 5}$ & $\mathbf{0 . 1 4}$ & $\mathbf{0 . 2 6}$ & $\mathbf{0 . 3 9}$ & $\mathbf{0 . 5 3}$ & $\mathbf{0 . 8 3}$ \\
SBS SyG & $\mathbf{1 2 0}$ & $\mathbf{0 . 0 0 8}$ & $\mathbf{0 . 0 1 4}$ & $\mathbf{0 . 0 2 8}$ & $\mathbf{0 . 0 5 3}$ & $\mathbf{0 . 0 8 5}$ & $\mathbf{0 . 0 1 2}$ \\
\hline
\end{tabular}

The SBS UVX galaxies comprise $\sim 12 \%$ of field galaxies, and around $10 \%$ of them are Sy galaxies. The proportion of the SBS ELG without UV excess is a little less than the proportion of SBS UVX galaxies. Together they bring the number of ELG with strong emission to no less than $20 \%$ of field galaxies.

120 SBS Sy galaxies were found; 62 classical and 58 non-classical Sy galaxies. The proportion of SBS SyG among the field galaxies in a volume of about $500 \mathrm{Mpc}$ radius is around $1.2 \%$, slightly greater than in a volume of $\sim 100 \mathrm{Mpc}$ radius, where the value of $\sim 1 \%$ was first obtained on the basis of the Markarian survey. In addition, with the nearly 100 LINERs discovered in the SBS, AGN comprise no less than $2 \%$ of field galaxies. The redshift distribution of the total SBS galaxy sample is shown in Fig.1a.

A complete sample of 71 new faint SBS Sy galaxies in the magnitude range $13.0<B \leq 17.0$ with redshift $z \leq 0.1$ was isolated. It included: 40 classical Sy galaxies (17 Sy1, $5 \mathrm{Sy} 1.5,18 \mathrm{Sy}$ ) and 31 non-classical Sy galaxies (15 NLSy1, 16 transition (Sy2/LINER) objects). We were not able to classify the remainder 
of the objects with the use of the standard classification scheme, where the line width and the line ratio are used, therefore we include them in the class of non-classical Sy galaxies.

Numerous non-classical Sy galaxies were found in the last decade among the ROSAT and IRAS optically identified objects. The subsample of so-called NLSy1 is dominant among the ROSAT detected AGNs. The subsample of nonclassical Sy2 and Sy2/LINER composites is detected among the IRAS AGNs. These objects show emission line widths and emission line ratios not typical for classical Sy galaxies.

The subsample of SBS NLSy1 galaxies shows line ratios of $[O I I I] \lambda 5007 / H \beta$ from 0 to 10 , and FWHM line widths from $300-3000 \mathrm{~km} \mathrm{~s}^{-1}$, which fills the gap between Sy2s and BLSy1s. Some of the SBS Sy1 galaxies show very broad (or multicomponent) $H \alpha$ emission lines along with an absence of $H \beta$ or other emission lines. Some SBS Sy2s show narrow emission lines $H \alpha$ and $[N I I] \lambda 6584$, with $H \alpha /[N I I] \lambda 6584 \sim 1$, but $H \beta$ or $[O I I I] \lambda 5007$ are very weak or not seen at all. Some objects show the signature of composite Sy2/LINER spectra or LINER/Starburst spectra. There is continuity from classical BLSy1s through NLSy1s, Sy2s, through LINERs and Starbursts.

\subsection{The Complete Sample of SBS QSOs. Magnitude Range $15.7<B$ $\leq \mathbf{1 7 . 5}$, Redshift Range $0.3 \leq \mathrm{z} \leq \mathbf{2 . 2}$}

It is well known that in an optical UVX survey, the waveband $3500-5400 \AA$ is used for AGN/QSO detection, where selection effects are minimized in the redshift range $0.3<z<2.2$. The complete sample of SBS QSOs in the redshift range $0.3 \leq z \leq 2.2$ and magnitude range $15.7<B \leq 17.5$ contains $\sim 130$ objects. The redshift distribution of the total and complete samples of SBS AGN/QSOs is shown in Fig. 1b.

As in the case of SBS Sy galaxies, the complete sample of SBS QSOs also might be roughly divided into two groups: QSOs with Broad emission lines $F W H M>3000 \mathrm{~km} \mathrm{~s}^{-1}$ (BLQSOs) and QSOs with relatively narrow emission lines $F W H M<3000 \mathrm{~km} \mathrm{~s}^{-1}$ (NLQSOs or QSO II).

It seems to us that the NLQSOs are the continuation of NLSy1 to the class of more luminous objects, the so-called QSO IIs. Examples of similar objects were recently found among the IRAS selected ROSAT galaxies (Morgan et al. 1996). The spectra of some SBS NLQSOs are shown in Fig. 2.

The differential surface density of bright SBS QSOs in the magnitude ranges $16.0<B<16.5,16.5<B<17.0$ and $17.0<B<17.5$ is $0.016,0.34$, and 0.71 , respectively. The $\operatorname{LogN}-\mathrm{B}$ relation of SBS QSOs is $\log N-B=0.65 \pm 0.004$ in the magnitude range $15.7<B \leq 17.5$.

\section{Multiwavelength AGN (QSOs+SyG) Survey in the SBS Area in $\mathrm{X}$-ray, Optical, Infrared and Radio Wavebands}

In past decades we were able to work only in the optical window. As a result, the majority of observational data and cosmological models were based on the results of optically complete samples of AGN/QSOs. The first sample of AGNs (Markarian survey) and the first sample of bright QSOs (BQS, Schmidt \& Green 1983) have been used as a basis for cosmological calculation until now. During 


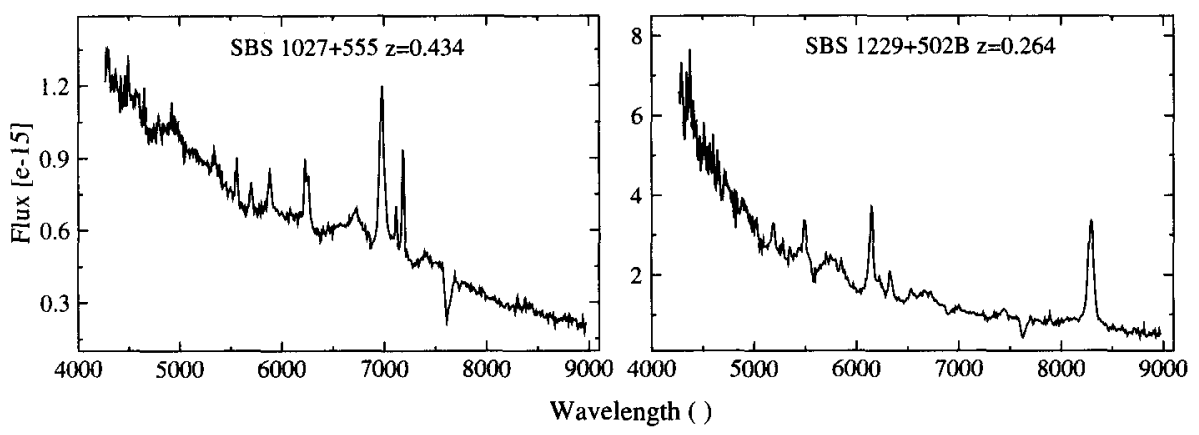

Figure 2. Examples of Narrow-Line SBS QSOs (QSO IIs).

the last two decades, these surveys were re-examined many times in regard to their completeness.

In the last decade, the IRAS, ROSAT, NVSS, FIRST, 2dF, SDSS and 2MASS data completely changed the astronomical database. A huge number of new X-ray, IR, radio, etc, AGN/QSOs were discovered, many of which were not present in optical complete samples. Of course, the combination of different selection techniques will eventually produce a more complete flux limited sample of AGN/QSOs than a single parameter survey.

The multiwavelength AGN survey (MWAGN), aimed for the construction of a combined deep sample of AGNs based on the SBS survey in X-ray, optical, IR and radio wavebands, was undertaken in the last few years. We have used the complete sample of SBS optically selected AGNs as the basis for the compilation of a combined multiwavelength complete sample of bright AGN/QSOs. It is the first attempt to create a sample of AGN/QSOs with a well defined flux limit, where a wide range of the electromagnetic spectrum is used. Nearly 15000 optical counterparts of ROSAT, FIRST and IRAS sources were identified to the magnitude limit of DSS1. A few thousand relatively bright objects with optical counterparts with $B \leq 17.5$ were isolated for spectoscopic observation.

The subsample of optically bright $B \leq 17.5$ and ROSAT Bright $\left(f_{x}>2.4 \times\right.$ $\left.10^{12} \mathrm{erg} \mathrm{cm}^{-2} \mathrm{~s}^{-1}\right)$ sources in the SBS area were spectroscopically completely investigated. A dozen ROSAT and optically bright new AGN/QSOs were found.

The Radio-Optical survey in the SBS area was undertaken by V.Chavushyan. FIRST sources were indentified with APM stellar optical counterparts, the bright part of which $(B \leq 17.5)$ was spectroscopically completely investigated. A sample of about 30 optically bright $(B \leq 17.5)$ new AGN/QSOs was discovered. The preliminary result of the multiwavelength FIRST/APM QSO survey in the SBS area was published by Chavushyan et al. (1999, 2001).

The investigation of Infrared sources in the SBS area is underway on the SPM (Mexico) 2.1-m telescope (I.Cruz-Gonzalez, D.Dultzin-Hacyan).

Preliminary results of the MWAGN survey in the SBS area are presented in Table 3.

Twelve new bright $(\mathrm{B} \leq 17.5)$ QSOs with redshifts $0.3 \leq \mathrm{z} \leq 2.2$ which are ROSAT and/or FIRST sources were discovered in the course of the MWAGN survey in the SBS area. The resulting $\log N-B$ relation becomes $\log N-B=$ 
Table 3. The complete sample of SBS QSOs and QSOs of the MWAGN survey in the SBS area. Magnitude range $16.0<\mathrm{B} \leq 17.5$, redshift range $0.3<\mathrm{z} \leq 2.2$

\begin{tabular}{|c|c|c|c|c|c|c|c|c|}
\hline & \multicolumn{3}{|c|}{ SBS Optical survey } & \multicolumn{2}{|c|}{ ROSAT } & \multicolumn{2}{|c|}{ FIRST } & MWAGN \\
\hline \multirow{2}{*}{$\begin{array}{l}\text { Mag. } \\
\text { range }\end{array}$} & $\mathbf{N}$ & \multicolumn{2}{|c|}{ Missed in SBSS } & $\mathbf{N}$ & Detected & $\mathbf{N}$ & Detected & Total \\
\hline & Tot. & ROSAT & FIRST & Tot. & in SBS & Tot. & in SBS & number \\
\hline $16.0-16.5$ & 16 & - & $\mathbf{1}$ & 2 & 2 & $\overline{3}$ & 2 & 17 \\
\hline 17.0 & 34 & 1 & 2 & 11 & 10 & $\mathbf{5}$ & 3 & 37 \\
\hline $17.0-17.5$ & 71 & 3 & $\mathbf{5}$ & 15 & 12 & 15 & 10 & 79 \\
\hline & $\log 1$ & $\overline{0.65} \pm 0$. & $4+C$ & & & $=0$. & $0.005+C$ & \\
\hline
\end{tabular}

$0.67 \pm 0.005$. Of course, this magnitude range is too narrow to make the critical cosmological conclusion about the behavior of the LogN-B curve, but the MWAGN data may allow us to conclude that the fast evolution of bright QSOs is more likely the result of selection effects than the result of cosmological evolution.

The effect of overlap is empirically obtained. The MWAGN data allow us to make a direct estimate. Part of the AGN/QSOs missed in the SBS which are ROSAT or FIRST sources are in fact AGNs contaminated by nearby objects. For objects brighter than $B \leq 17.0$ the effect of overlap is $\sim 5 \%$; for $B \leq 17.5$ magnitude the effect of overlap is $\sim 10 \%$.

The MWAGN survey in the SBS area may allow us to compile a complete sample of AGN/QSOs with a well defined flux limit: in the optical range $B \leq$ 17.5 , in the soft $(0.5-2 \mathrm{keV}) \mathrm{X}$-ray band $f_{x}>2.4 \times 10^{-12} \mathrm{erg} \mathrm{cm}{ }^{-2} \mathrm{~s}^{-1}$, in the IRAS band $f(60 \mu)>0.6$ Jy and Radio fluxes at $1.4 \mathrm{GHz}$ greater than $1 \mathrm{mJy}$.

The MWAGN survey in the SBS area is not finished yet, and the combined complete sample of AGN/QSOs in the SBS area is not compiled yet, but a few important conclusions might be drawn from the current data:

1. On the basis of the Second Byurakan Survey (SBS) we have produced the largest and most homogeneous new complete samples of faint Markarian Galaxies, Sy galaxies and bright QSOs.

2. The volume of reliable investigation of UVX galaxies, extended out to a distance of $\sim 500 \mathrm{Mpc}$, is more than 50 times deeper than in the FBS. The proportion of UVX galaxies among field galaxies in the volume of about $500 \mathrm{Mpc}$ is about $12 \%$, which is slightly greater than in the FBS survey $(\sim 100 \mathrm{Mpc}, 10 \%)$.

3. The first complete sample of faint $\left(B \leq 17^{\mathrm{m}} 0, z<0.1\right)$ Sy galaxies is compiled. The relative number of SBS SyG consists of about $10 \%$ of SBS faint UVX galaxies or $1.2 \%$ of field galaxies. A complete sample of relatively bright SBS QSOs in the magnitude range $15.7 ; \mathrm{B} \leq 17.5$ and redshift range $0.3 \leq \mathrm{z} \leq 2.2$ was constructed as well .

4. The existing classification of AGN/QSOs is not sufficient for the classification of the entire class of newly detected AGN/QSOs. The sample of SBS AGN/QSOs might be divided into two groups: classical AGN/QSOs, consistent with the previous classification 
scheme and non-classical AGN/QSOs, which needed new classification criteria. The presence of narrow line QSOs (NLQSOs) or so-called QSO II type objects is established. Narrow Line QSOs are the continuation of NLSy1 to the class of more luminous objects (QSO II class) .

5. There is continuity from classical BLSy1s through NLSy1s, Sy2s, to LINERs and Starbursts. The subsample of SBS NLS1 galaxies shows line ratios of $[\mathrm{OIII}] / \mathbf{H} \beta$ from 0 to 10, and FWHM line widths from $300-3000 \mathrm{~km} \mathrm{~s}^{-1}$, which fills the gap between Sy2s and BLSy1s.

6. The preliminary result of the Multiwavelength AGN survey in the SBS area shows that the ROSAT, FIRST or IRAS surveys alone cannot represent the whole AGN sample. Only 25\% of SBS QSOs are ROSAT or FIRST sources. The IRAS survey is effective for redshifts less than $z<0.05$. All classical Sy2s, but only $\sim 50 \%$ of classical Sy1s are IRAS sources. Luminous and Ultraluminous IRAS SBS AGNs comprise $\sim 25 \%$ of the whole AGN sample. Most SBS AGN/QSOs are neither ROSAT nor FIRST nor IRAS LIG or ULIG sources. They are optically emitting AGN/QSOs.

7. The multiwavelength complete sample of bright SBS QSOs allows us to conclude that the fast evolution of bright QSOs is more likely the result of selection effects than the result of cosmological evolution.

Acknowledgments. J.S. is grateful to the Organizing Committee especially to the chairman of the Local Organizing Committee, A. Mikaelian, and to UNAM colleagues, D. Dultzin-Hacyan, I.Cruz Gonzalez and Rafael Costero through the help of whom I had the possibility to participate in the Colloquium. We extend our thanks to the Byurakan co-workers and high quality technical specialists without whom the SBS couldn't be arranged and continued: Aram Astvazaturian (Varpet Aram), S.Karapetian, H. Sarkisian and others.

J.S. thanks G. Kojoian, D. Weedman and R. West who provided the IIIaJ plates at the beginning of the SBS survey. We thank Maarten Schmidt who in 1986 arranged the investigation of the SBS objects with R. Green and C. Foltz. Thank to R. Green and C. Foltz, many of the SBS objects were investigated in more detail. Thanks to Donald Osterbrock and his collaborators, the investigation of the SBS Sy galaxies was continued.

Thanks to V. Afanasév, we had a chance to continue our work in the Special Astrophysical observatory (Russia). Due to the help of Irene Cruz Gonzalez and Deborah Dultzin-Hacyan, the investigation of SBS objects are continuing now in UNAM (Mexico).

J.Stepanian acknowledges the CONACYT Catedras Patrimoniales project EXP.EX-000287. V.Chavushyan acknowledges CONACYT research grant 28499E. 


\section{References}

Chavushyan V.H., et al., 2001, ASP. Conf.Ser. 102, 232, Ed. by Clowes et al. Markarian, B.E., 1967, Astrofizika, 3, 55

Markarian, B.E., et al. 1981, Astrofizika, 17, 619

Markarian, B.E., \& Stepanian, J.A., 1983, Astrofizika, 19, 639

Markarian, B.E., \& Stepanian, J.A., 1984a;b, Astrofizika, 20, 21, 513

Markarian, B.E., Stepanian, J.A., \& Erastova, L.K. 1985, Astrofizika, 23, 439

Markarian, B.E., Stepanian, J.A., \& Erastova, L.K. 1986, Astrofizika, 25, 345

Morgan et al. 1996, ApJS, 106, 341

Schmidt, M. \& Green, R.F., 1983, Ap.J., 269, 352.

Stepanian, J.A., Lipovetsky, V.A., \& Erastova, L.K., 1988, Astrofizika, 29, 247

Stepanian, J.A., Lipovetsky, V.A., \& Erastova, L.K., 1990a, Astrofizika, 32, 441

Stepanian, J.A., Lipovetsky, V.A., Shapovalova, A.I., \& Erastova, L.K., 1990b, Astrofizika, 33, 89

Stepanian, J.A., Lipovetsky, V.A., Shapovalova, A.I., Erastova, L.K., \& Chavushyan, V.H., 1990c;d; Astrofizika, 33, 199, 351

Stepanian, J.A., Lipovetsky, V.A., Chavushyan, V.H., Erastova, L.K., \& Shapovalova, A.I., 1991a;b; Astrofizika, 34, 205, 315

Stepanian, J.A., Lipovetsky, V.A., Chavushyan, V.H., Erastova, L.K., \& Balayan, S.K., 1993, Bull. Spec. Astrophys Obs. (Izv. SAO), 36, 5

Stepanian, J.A., Chavushyan, V.H., Carrasco, L., Tovmassian, H.M., \& Erastova, L.K., 1999, PASP, 111, 1099

Stepanian, J.A., 1994, Doctoral thesis, Nizhnij Arkhys

Stepanian, J.A, IAU Symp 194 "Activity in Galaxies and related phenomena" 1998 Kluwer Acad. Publ. P.O.Box 17, 3300 AA Dordrecht, The Netherlands 